\title{
Modeling Interplanetary Logistics: A Mathematical Model for Mission Planning
}

\author{
Christine Taylor, ${ }^{*}$ Miao Song, ${ }^{\dagger}$ Diego Klabjan ${ }^{\ddagger}$ \\ Olivier L. de Weck ${ }^{\S}$ and David Simchi-Levi ${ }^{\Uparrow}$ \\ Massachusetts Institute of Technology, Cambridge, MA 02139
}

\begin{abstract}
The objective of this paper is to demonstrate a methodology for designing and evaluating the operational planning for interplanetary exploration missions. A primary question for space exploration mission design is how to best design the logistics required to sustain the exploration initiative. Using terrestrial logistics modeling tools that have been extended to encompass the dynamics and requirements of space transportation, an architectural decision method has been created. The model presented in this paper is capable of analyzing a variety of mission scenarios over an extended period of time with the goal of defining interesting architectural scenarios for space logistics. This model can be utilized to evaluate different logistics trades, such as a possible establishment of a push-pull boundary, which can aid in commodity pre-positioning. The results of this implementation are presented for a lunar campaign using estimated surface demands for exploration.
\end{abstract}

\section{Introduction}

$\mathrm{O}$ January 14th, 2004, President Bush set forth a new exploration initiative to achieve a sustained human presence in space. Included in this directive is the return of humans to the Moon by 2020 and the human exploration of Mars thereafter. ${ }^{1}$ The President has tasked NASA with the development of a sustainable space transportation system that will enable continual exploration of the Moon, Mars and 'beyond'.

Inherent to the problem of transporting people to the Moon, Mars, and 'beyond' is sustaining the people and the operations while in transit and at the respective destinations. Especially for long-term missions, the amount of consumables required becomes a significant issue in terms of mass in Low Earth Orbit (LEO) which translates to mission cost. In order to develop a sustainable space transportation architecture it is critical that interplanetary supply chain logistics be considered.

The goal of the interplanetary supply chain logistics problem is to adequately account for and optimize the transfer of supplies from Earth to locations in space. Although the commodities themselves may be of low value on Earth, the consideration of these commodities is of high importance and can directly impact the mission success. As such, it is desirable to find low cost yet reliable methods of transporting these supplies to their destinations.

The space exploration missions will evolve over time, which will generate an increased demand at in-space locations. In order to develop a sustainable architecture it is necessary to recognize the interdependencies between missions and how this coupling could affect the logistics planning. By viewing the set of missions together, as a space network, and optimizing the operations of the transportation system that provides the logistics for the exploration missions, a reduction in cost can be achieved which promotes a more sustainable system architecture.

\footnotetext{
*Graduate Student, Department of Aeronautics and Astronautics, 77 Massachusetts Avenue, Room 33-409, c_taylor@mit.edu, AIAA Student Member

†Graduate Student, Department of Civil and Environmental Engineering, 550 Memorial Drive, Apt 16 F, msong@mit.edu,

$\ddagger$ Visiting Professor, Depratment of Civil and Environmental Engineering, 77 Massachusetts Avenue, Room 1-174, klabjan@mit.edu

$\S$ Assistant Professor, Department of Aeronautics and Astronautics, Engineering Systems Division, 77 Massachusetts Avenue, Room 33-412, deweck@mit.edu, AIAA Senior Member

『Professor of Engineering Systems Division, 77 Massachusetts Avenue, Room 1-171, dslevi@mit.edu
} 
There exists a great deal of literature on the design of transportation networks on Earth. For example in Reference 2 the design of the school bus routing problem is presented and solved. In this problem there exists a number of restrictions on feasible solutions, including time window constraints on pick-up and delivery, which add to the complexity of a large-scale problem. In Reference 3 a smaller aircraft network design problem is considered to understand the effects of the network design and vehicle selection on the system cost. By defining three different classes of aircraft, small, medium, and large, the optimal allocation of vehicles to routes can be defined to meet the given demand.

Many of the tools and methods of terrestrial logistics can be extended to space networks. Specifically, time expanded networks represent a method for modeling transportation systems that are operated over time. ${ }^{4}$ Using this modeling technique the static network is expanded and time is incorporated directly into the network definition. As shown in Reference 5 time expanded networks were used to plan the routing of trucks for companies that rely on less than truck load carriers for shipping products to customers.

The development of an interplanetary supply chain requires the unification of two traditionally separate communities: aerospace engineering and operations research. Since these two communities have traditionally operated independently, separate terminologies for describing components of the problem have been developed. Therefore, in order to create an effective means of communication between all members involved, a distinct terminology has been developed. In Section II, this terminology is detailed extensively. Specifically, the definition of the commodities or supplies and the elements or physical containment and propulsion units used to transport the commodities. Furthermore, the network definition is presented as well as the definition and description of the time expanded network which is the terrestrial modeling technique employed for the space logistics model. Section III presents the problem formulation and constraints. In Section IV, a description of the optimization methodology developed to solve this problem is discussed. The problem formulation and solution methodology is applied for the example of a lunar campaign, and the results of this implementation are presented in Section V. Section VI reviews the contributions of this paper and describes continuing work in this area.

\section{Problem Definition}

The first step in developing a model for interplanetary logistics is defining a concrete nomenclature that describes the components of the problem. The problem fundamentally consists of three components: the commodities or supplies that must be shipped to satisfy a mission demand, the elements or physical structures used to both hold and move the commodities, and the network or pathways the elements and commodities travel on. The following sub-sections define the parameters that describe each of these components.

\section{A. Commodities}

The goal of the space logistics project is to determine how to meet the demand for the exploration missions. As such, we are investigating how to optimally ship multiple types of commodities. For the purpose of the logistics problem, a commodity will be defined as a high-level aggregate of a type of supply, such as crew provisions. Thus, we will define a set of $k=1, \ldots, K$ commodities, each with the following parameters.

- Denote the demand of each commodity as $d^{k}$.

- Denote the origin of each commodity as $s o^{k}$.

- Define the destination of each commodity as $s d^{k}$.

- Define the availability interval of each commodity as $t o^{k}=\left[s t o^{k}, e t o^{k}\right]$, where $s t o^{k}$ is the starting time of the interval and $e t o^{k}$ is the ending time of the interval.

- Define the delivery interval of each commodity as $t d^{k}=\left[s t d^{k}\right.$, et $\left.d^{k}\right]$, where $s t d^{k}$ is the starting time of the interval and $e t d^{k}$ is the ending time of the interval.

- Define the maximum time that a commodity can be in transit as $t_{\text {max }}^{k}$.

- Define the unit mass of each commodity as $m^{k}$ when it arrives at the destination.

- Define the unit volume of each commodity as $v^{k}$ when it arrives at the destination. 
- Define an absolute mass gain/loss factor for each commodity after being available at $s o^{k}$ for $\tau$ periods as $f m_{\tau}^{k}$ where $f m_{\tau}^{k}<0$ if the commodity gains mass over time, $f m_{\tau}^{k}>0$ if the commodity loses mass over time and $f m_{\tau}^{k}=0$ if the commodity mass remains constant over time. $a$

- Define an absolute volume gain/loss factor for each commodity after being available at $s o^{k}$ for $\tau$ periods as $f v_{\tau}^{k}$ where $f v_{\tau}^{k}<0$ if the commodity gains volume over time, $f v_{\tau}^{k}>0$ if the commodity loses volume over time and $f v_{\tau}^{k}=0$ if the commodity volume remains constant over time.

It is important to note that in this model a crew member is treated as a commodity. In practice, crewed missions are treated differently during mission planning, however, for the purposes of the architectural design tool created by this model, crew can be considered a commodity with highly restrictive parameter values. By narrowing the availability and delivery windows for a crew commodity, the feasible shipment pathways are limited and reasonable architectures for crewed flights can be obtained.

\section{B. Elements}

In order to ship the commodities from the origin to the destination locations, we require both containers to hold the commodities and propulsion to move the mass through space. These components can be abstracted to a single definition of an element. Elements are physical, indivisible functional units that transport the commodities from origin to destination. An element is classified by the amount of commodity capacity and propulsive capability it possesses. Elements can be divided into two classes: non-propulsive elements $\mathcal{M}_{N}$ and propulsive elements $\mathcal{M}_{P}$. The element parameters are (cf. Figure 1) as follows.

- The maximum fuel mass of a propulsive element $m, m \in \mathcal{M}_{P}$ is denoted by $m f^{m}$.

- The fuel volume of a propulsive element $m$ is denoted by $v f^{m}$.

- The structural mass of element $m$ is denoted by $m s^{m}$.

- The mass capacity of element $m$ is denoted by $C M^{m}$.

- The volume capacity of element $m$ is denoted by $C V^{m}$.

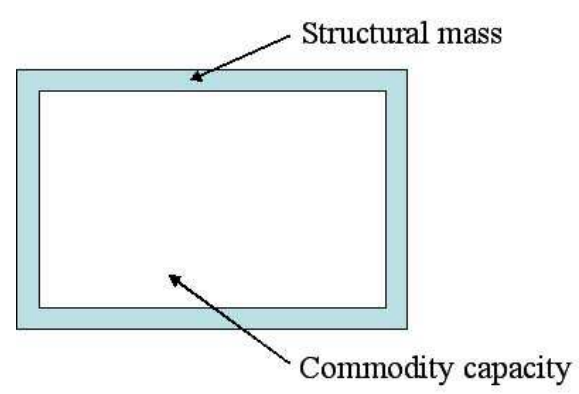

(a) Non-propulsive element

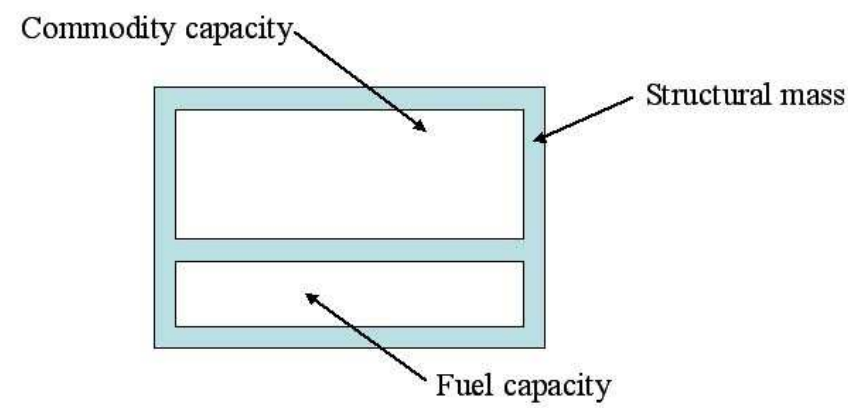

(b) Propulsive element

Figure 1. Element Representation

\section{Networks}

In order to transfer the commodities and elements from the origin node to the destination node, the trajectories must be defined. The purpose of the interplanetary logistics model developed in this paper is to analyze the multiple choices available for routing all of the commodities and elements to determine the best logistics architecture. To model the different available trajectories, a network model of space is created to represent the possibilities available for transferring commodities to their respective destination. The following subsections detail the development of the space network utilized to form the model presented in this paper. a For example, let commodity $k$ become available at its origin $s o^{k}$ at time $t_{o} \in t o^{k}$ and arrive at the destination $s d^{k}$ at time
$t_{d} \in t d^{k}$. For any time $t_{c} \in\left[t_{o}, t_{d}\right]$, the unit mass of commodity $k$ at time $t_{c}$ is $m^{k}+\sum_{t=t_{c}}^{t_{d}} f m_{t-t_{o}}^{k}$. 


\section{Static Network}

The physical network, or static network, represents the set of physical locations, or nodes, and the connections, or arcs, between them. The physical nodes, or static nodes, represent the different physical destinations in space, including the origin and destination of all the commodities, as well as the possible locations for transshipment. Three types of nodes have been identified: Body nodes, Orbit nodes, and Lagrange point nodes. The physical arcs, or static arcs, represent the physical connections between two nodes, that is, an element can physically traverse between these two nodes. We define an arc $(s i, s j)$ to be a static arc that represents a feasible transfer from static node si to static node $s j$.

The mathematical description of the static network is given below.

- Define the static network as a graph $G S$, where $G S=(N S, A S)$.

- Define the set of nodes, $N S=\{s 1, \ldots, s n\}$, in the static network.

- Define the set of arcs, $A S \subseteq N S \times N S$ in the static network.

An example of an Earth-Moon static network is provided in Figure 2. In this picture, we can see the connection of the Earth surface nodes to the Earth orbit nodes, representing launches and returns. Similarly, the lunar surface nodes are connected to the lunar orbit nodes, representing descent and ascent trajectories. In addition, the orbit nodes, as well as the first Earth-Moon Lagrangian point are connected by in-space trajectories.

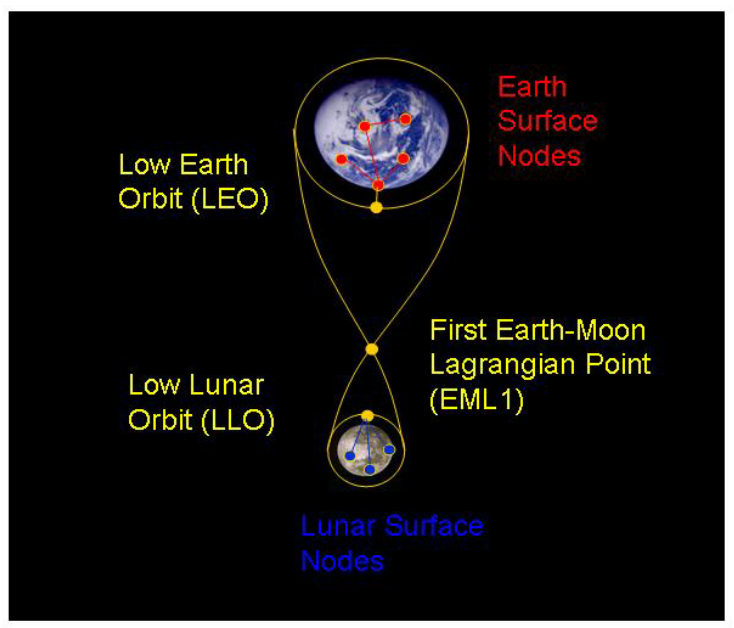

Figure 2. Depiction of an Earth-Moon Static Network

\section{Time Expanded Network}

The space logistics project is investigating the design of a sequence of missions that evolve over an extended period of time. In addition, certain properties of the space network are time-varying. For these reasons we have chosen to introduce time expanded networks as a modeling tool. In the time expanded network, the absolute time interval under consideration is discretized into $T$ time periods of length $\Delta t$. A copy of each static node is made for each of the time points and the nodes are connected according to the following rules.

- The arc must exist in the static network.

- The arc must create a connection that moves forward in time.

$$
4 \text { of } 15
$$


- The arc must represent a feasible transfer, in terms of orbital dynamics.

The mathematical description of the time expanded network is given below.

- Define the time expanded network as a graph $\mathcal{G}$, where $\mathcal{G}=(\mathcal{N}, \mathcal{A})$.

- Define the set of nodes in the time expanded network as $\mathcal{N}=\{i=($ si,t $) \mid$ si $\in N S, t=1, \ldots, T\}$. To simplify the notation, for a given node $i \in \mathcal{N}$, let $s(i)$ and $t(i)$ denote the physical node and the time period corresponding to node $i$, i.e., if $i=(s i, t)$ then $s(i)=s i$ and $t(i)=t$.

- Define node $s$ as the general source that generates the supply of elements. This node is connected to every node in the network where an element can originate (e.g. in the current setting $s$ is connected to every node $i$ with $s(i)$ corresponding to Low Earth Orbit (LEO)).

- Define the set of arcs in the time expanded network as $\mathcal{A} \subseteq \mathcal{N} \times \mathcal{N}$. An arc $a=(i, j)=((s i, t),(s j, t+$ $\left.\left.T_{s i, s j}^{t}\right)\right)$ exists if and only if there exists an arc $(s i, s j)$ in the static network, and the transit time from static node $s i$ to static node $s j$ starting at time $t$ is $T_{s i, s j}^{t}$. Note that if $s i=s j$, then $T_{s i, s j}^{t}=1$ for all $t$.

- Define path $p$ as a sequence of nodes. In particular, let $f(p)$ and $l(p)$ denote the first node and the last node of path $p$. If path $p$ originates at node $s, f(p)=s$ for all such $p$.

Using the static network depicted in Figure 2, we can create the time expanded network in Figure 3. Here, the time expanded network is notional as not all arcs are represented, but how the trajectories evolve in time can be readily seen.

day 1

day 2

day 3

day 4

day 5

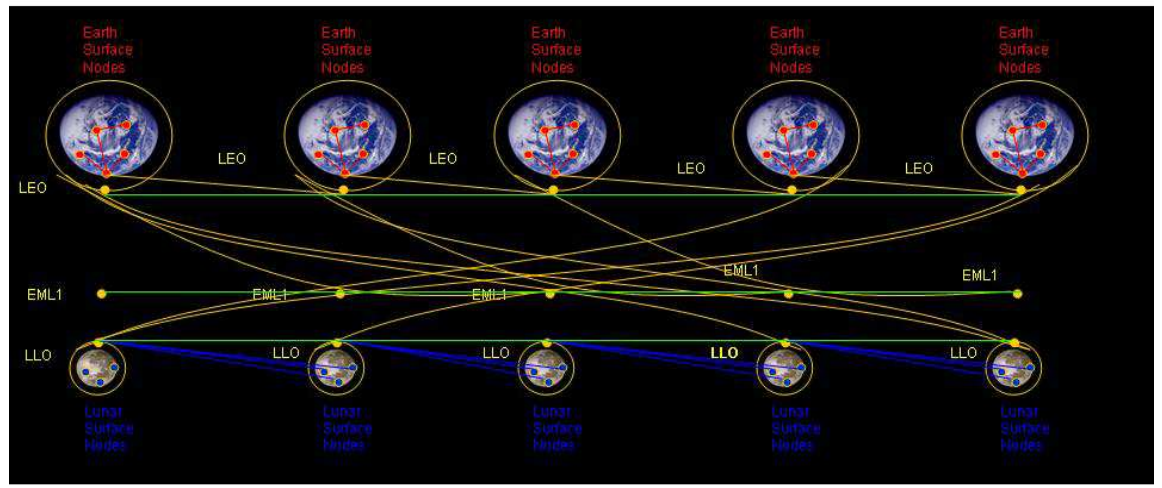

Figure 3. Depiction of an Earth-Moon Time Expanded Network

To account for the fact that on certain transfer arcs two burns occur, we slightly modify the time expanded network. We first introduce a new fictitious static node labeled fic. Note that this node is not related to the static network. On every transfer arc $(i, j), s(i) \neq s(j)$ requiring two burns we add a new auxiliary node $k=($ fic,$t)$ with two arcs; one connects $i$ to $k$ and the other one $k$ to $j$. The value of $t$ is irrelevant. In this new network, each arc $(i, j)$ with $s(i) \neq s(j)$ corresponds to a single burn. All such arcs are called burn arcs and we denote them by $\mathcal{A}_{B}$.

The mass fraction for element $m$ to execute the burn corresponding to arc $a \in \mathcal{A}_{B}$ is defined as

$$
\phi_{a}^{m}=1-\exp \left(\frac{-\Delta V_{a}}{I^{m} g_{0}}\right) .
$$

$$
5 \text { of } 15
$$


which is taken from the rocket equation. ${ }^{6}$

\section{Formulation}

Having defined the network, commodities, and elements, the interplanetary logistics model can be presented. The model is developed in three stages. First, the flow of commodities is defined and the constraints governing the commodity flows are presented. Next, the element flows are modeled with the corresponding constraints. Finally, the constraints governing the capacity and capability, which represent the coupling constraints between the commodities and elements are developed.

\section{A. Assumptions}

In order to define the mathematical model for interplanetary logistics, the modeling assumptions used during the model definition are presented. The following assumptions about the behavior of elements are made to create a computationally tractable model.

Consecutive Burns An active element burns only on consecutive burns. Once an element becomes active, it stays active for a certain number of burns. As soon as it becomes passive, it can no longer be active again unless it is refueled. Between two consecutive burns, an active element can be idle for an arbitrary length of time. The number of consecutive burns is not constrained.

Fuel Consumption We assume that before every initial burn, the active element is filled to capacity with fuel and after the burns are completed, the remaining fuel is expelled. If an element is later refueled, it is filled to maximum capacity.

For example, consider an element that starts burning. Just before this first burn the element was filled to capacity with fuel. The element then executes four consecutive burns and after the fourth burn it expels any remaining fuel. Then it travels as a passive element for a period of time. If at some point it is refueled, it can remain passive for another period of time before it executes another sequence of burns.

Docking/Undocking We assume that any two elements can be docked and undocked. In addition, if any cost is associated with these operations, it is not explicitly captured. If some elements cannot be docked together, then this must be captured in a post optimization analysis.

In-Space Modeling This model represents the in-space transportation model beginning at LEO and therefore does not capture launching. Although launching is an important component of logistics design, the constraints are not well represented in the time-expanded network model. Instead, a separate launching model must be created to examine packing as well as scheduling constraints. Finally, since many mission architectures launch to LEO before preceding to in-space destinations, LEO represents a good point for decoupling the launch decisions from the in-space network decisions.

\section{B. Commodity Flows}

\section{Commodity Path Feasibility}

In order to understand how each commodity should move through the network it is not sufficient to know which arcs are traversed. Instead, it is necessary to determine the path followed from the origin node to the destination node where the commodity fulfills the specified demand. If we define a path variable $p$, then for each commodity $k$ it is possible to determine a set of feasible paths $\mathcal{P}^{k}$.

For a given commodity $k$, the path $p$ is feasible only if it originates at node $i=\left(s o^{k}, t\right)$ with $t \in t o^{k}$ and terminates at node $j=\left(s d^{k}, t^{\prime}\right)$ with $t^{\prime} \in t d^{k}$. Moreover, we require that the transit time along the path $p$ is no greater than the maximum travel time for commodity $k$, i.e.,

$$
t(l(p))-t(f(p))=\sum_{(i, j) \in p}(t(j)-t(i)) \leq t_{\max }^{k} \quad p \in \mathcal{P}^{k}
$$




\section{Commodity Flow Variables and Constraints}

We need to determine how many units of commodity $k$ are transported on path $p$, for any $k$ and $p \in \mathcal{P}^{k}$. Therefore, for every $k$ and $p \in \mathcal{P}^{k}$ we have a decision variable $x_{p}^{k} \geq 0$ such that

$$
x_{p}^{k}=\text { number of units of commodity } k \text { traveling on path } p .
$$

In order to satisfy the demand $d^{k}$ of a given commodity $x_{p}^{k}$, we have

$$
\sum_{p \in \mathcal{P}^{k}} x_{p}^{k}=d^{k} \quad \text { for every commodity } k .
$$

\section{Element Flows}

\section{Element Flow Variables}

For any non-propulsive element $m \in \mathcal{M}_{N}$, let us define the decision variable $y_{p}^{m}$ such that

$$
y_{p}^{m}= \begin{cases}1 & \text { if non-propulsive element } m \text { travels on path } p \\ 0 & \text { otherwise }\end{cases}
$$

for each feasible path $p$ in the time expanded network.

Moreover, for any propulsive element $m \in \mathcal{M}_{P}$,

$$
z_{p, q}^{m}= \begin{cases}1 & \text { if element } m \text { is fueled at the first node of } p \text { and is active during sub-path } q \text { of path } p \\ 0 & \text { otherwise, }\end{cases}
$$

where $p$ is any feasible path in the time expanded network and $q$ is a sub-path of $p$. Note that $\sum_{q} z_{p, q}^{m}=1$ if and only if element $m \in \mathcal{M}_{P}$ travels on path $p$.

For each path $p$, the element $m$ can only be refueled at most once at the first node of $p$, and there is at most one sub-path $q$ such that the element $m$ is active. Note that some arc $a \notin \mathcal{A}_{B}$ may be included in the active sub-path $q$. It is possible for an element to enter the network without fuel, and be fueled at a node $i$. To capture this situation, we allow $q$ to be empty if $p$ is the first path of the element, i.e., the first node of $p$, $f(p)$ is $s$. As illustrated in Figure 4, this definition allows the tracking of refueling, and the active sub-path $q$ is empty for the first path $p_{0}$.

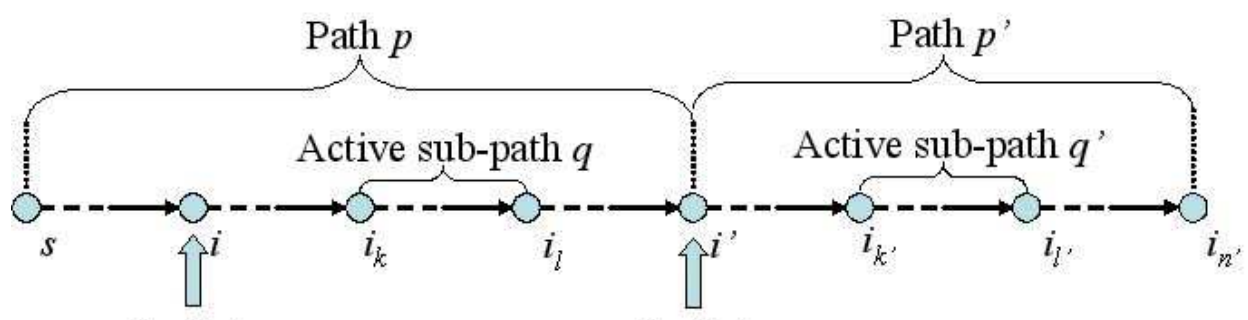

Fueled

Fueled

Figure 4. Illustration of the Propulsive Element Flow Variables

\section{Element Flow Constraints}

- A non-propulsive element can only travel on a single path,

$$
\sum_{p} y_{p}^{m} \leq 1 \quad m \in \mathcal{M}_{N}
$$

- For active elements, we constrain at most one element to be active on any burn arc,

$$
\sum_{m \in \mathcal{M}_{P}} \sum_{p} \sum_{q: a \in q} z_{p, q}^{m} \leq 1 \quad a \in \mathcal{A}_{B} .
$$

$$
7 \text { of } 15
$$


- A non-propulsive element $m \in \mathcal{M}_{N}$ can travel on an arc $a$ only if there is an active element on that arc,

$$
\sum_{p: a \in p} y_{p}^{m} \leq \sum_{m^{\prime} \in \mathcal{M}_{P}} \sum_{p} \sum_{q: a \in q} z_{p, q}^{m^{\prime}} \quad a \in \mathcal{A}_{B}, m \in \mathcal{M}_{N}
$$

- A propulsive element $m \in \mathcal{M}_{P}$ can travel on an arc $a$ only if there is an active element on that arc,

$$
\sum_{p: a \in p} \sum_{q} z_{p, q}^{m} \leq \sum_{m^{\prime} \in \mathcal{M}_{P}} \sum_{p} \sum_{q: a \in q} z_{p, q}^{m^{\prime}} \quad a \in \mathcal{A}_{B}, m \in \mathcal{M}_{P}
$$

- To obtain a valid formulation for refueling, we require the flow conservation constraints for each element $m \in \mathcal{M}_{P}$

$$
\begin{array}{rlrl}
\sum_{p: f(p)=s} \sum_{q} z_{p, q}^{m} & \leq 1 & m & \in \mathcal{M}_{P} \\
\sum_{p: l(p)=i} \sum_{q} z_{p, q}^{m} & =\sum_{p: f(p)=i} \sum_{q} z_{p, q}^{m} & m & \in \mathcal{M}_{P}, i \in \mathcal{N} .
\end{array}
$$

\section{Fuel Flows}

For every node $i$ where a propulsive element $m$ will be fueled, there should be a sufficient supply of fuel. From our assumptions it follows that the required amount of fuel be $m f^{m}$ for element $m$. Therefore, the amount of fuel required at node $i$ can be regarded as a special commodity, with the demand of $\sum_{m \in \mathcal{M}_{P}} \sum_{p: f(p)=i} \sum_{q} m f^{m} z_{p, q}^{m}$. For each of the feasible path $p$, we define the decision variable

$$
w_{p}=\text { amount of fuel traveling on path } p .
$$

Obviously, $w_{p} \geq 0$, and the demand of fuel at node $i$ is satisfied by the fuel transported on path $p$ ending at node $i$, i.e.,

$$
\sum_{p: l(p)=i} w_{p}=\sum_{m \in \mathcal{M}_{P}} \sum_{p: f(p)=i} \sum_{q} m f^{m} z_{p, q}^{m} \quad i \in \mathcal{N} .
$$

\section{E. Capacity}

For space travel, it is necessary that all commodities be transferred by elements. As such, we must relate the amount of commodities (both mass and volume) available at each time to the total capacity available at that time. Since the mass and volume loss/gain factors for different commodities can be both positive and negative, for each arc $a=(i, j)$, we consider the capacity at both $t(i)$ and $t(j) !$

First, for a given arc $a=(i, j)$, let us consider the mass capacity constraint at time $t(i)$.

- For any commodity $k$, for some path $p$ such that $a \in p$, we need to consider the mass of the amount $x_{p}^{k}$ at time $t(i)$. Commodity $k$ traveling along path $p$ enters the network at the first node of $p$, i.e., at time $t(f(p))$ and arrives at the destination at the last node of $p$, i.e., at time $t(l(p))$. According to our definition of the mass loss/gain factor, its mass at time $t(i)$ is $\left(m^{k}+\sum_{t=t(i)}^{t(l(p))} f m_{t-t(f(p))}^{k}\right) x_{p}^{k}$.

- We need to consider the fuel mass $w_{p}$ such that $a \in p$.

- The total mass capacity available at arc $a=(i, j)$ is

$$
\sum_{m \in \mathcal{M}_{P}} \sum_{p: a \in p} \sum_{q} C M^{m} z_{p, q}^{m}+\sum_{m \in \mathcal{M}_{N}} \sum_{p: a \in p} C M^{m} y_{p}^{m} .
$$

${ }^{\mathrm{b}}$ If we allow nonlinear loss/gain functions, we need to evaluate the capacity of arc $a$ at any time $t \in[t(i), t(j)]$. However, it is a direct extension of constraints discussed here. 
Therefore, the corresponding capacity constraint is

$$
\begin{aligned}
& \sum_{k} \sum_{p: a \in p}\left(m^{k}+\sum_{t=t(i)}^{t(l(p))} f m_{t-t(f(p))}^{k}\right) x_{p}^{k}+\sum_{p: a \in p} w_{p} \leq \sum_{m \in \mathcal{M}_{P}} \sum_{p: a \in p} \sum_{q} C M^{m} z_{p, q}^{m}+ \\
& \sum_{m \in \mathcal{M}_{N}} \sum_{p: a \in p} C M^{m} y_{p}^{m}
\end{aligned} \quad a=(i, j) \in \mathcal{A}_{B} .
$$

Similarly, we can get the mass capacity constraints at time $t(j)$,

$$
\begin{aligned}
& \sum_{k} \sum_{p: a \in p}\left(m^{k}+\sum_{t=t(j)}^{t(l(p))} f m_{t-t(f(p))}^{k}\right) x_{p}^{k}+\sum_{p: a \in p} w_{p} \leq \sum_{m \in \mathcal{M}_{P}} \sum_{p: a \in p} \sum_{q} C M^{m} z_{p, q}^{m}+ \\
& \sum_{m \in \mathcal{M}_{N}} \sum_{p: a \in p} C M^{m} y_{p}^{m} \quad a=(i, j) \in \mathcal{A}_{B} .
\end{aligned}
$$

As for volume capacity constraints, we have identical terms except for the fuel carried on arc $a$. The volume of the fuel traveled on path $p$ such that $a \in p$ is $\frac{v f^{m}}{m f^{m}} w_{p}$. Hence, the volume capacity constraints are

$$
\begin{array}{rl}
\sum_{k} \sum_{p: a \in p}\left(v^{k}+\sum_{t=t(i)}^{t(l(p))} f v_{t-t(f(p))}^{k}\right) x_{p}^{k}+\sum_{p: a \in p} \frac{v f^{m}}{m f^{m}} w_{p} & \leq \sum_{m \in \mathcal{M}_{P}} \sum_{p: a \in p} \sum_{q} C V^{m} z_{p, q}^{m}+ \\
\sum_{k} \sum_{p: a \in p}\left(v^{k}+\sum_{t=t(j)}^{t(l(p))} f v_{t-t(f(p))}^{k}\right) x_{p}^{k}+\sum_{p: a \in p} \frac{v f^{m}}{m f^{m}} w_{p} & \leq \sum_{m \in \mathcal{M}_{N}} C V^{m} y_{p}^{m} \sum_{m: a \in p} \sum_{q} C V^{m} z_{p, q}^{m}+ \\
\sum_{m \in \mathcal{M}_{N}} \sum_{p: a \in p} C V^{m} y_{p}^{m} & a=(i, j) \in \mathcal{A}_{B}
\end{array}
$$

\section{F. Capability}

The capability constraint determines if enough fuel is available to perform a burn. A single propulsive element can only burn on consecutive burn arcs. All fuel is assumed to be consumed or dropped after the final burn. The propulsive element cannot be reused until after it is refueled.

Here we model that the total fuel of the active element performing the burn on a sub-path $q$ must be enough to carry the total cumulative mass along every arc in $q$. Let $q$ be an arbitrary sequence of possible consecutive burns and let $a^{l}=\left(i^{l}, j^{l}\right)$ be the $l$ th burn arc in $q$ for $l=1, \ldots,|q|$. Here $|q|$ denotes the number of arcs in $q$. Let $r(p, q)$ denote the sub-path along path $p$ from the first node of $p$ to the first node of $q$, if $q$ is not empty. For example, $r(p, q)$ is the sub-path from node $i$ to node $i_{k}$ for the path $p$ shown in Figure 4 .

The resulting constraint family reads

$$
\begin{gathered}
m f^{m} \sum_{p} z_{p, q}^{m}+M\left(1-\sum_{p} z_{p, q}^{m}\right) \geq \sum_{l=1}^{|q|} \Phi_{q, l}^{m} \times\left[\sum_{m^{\prime} \in \mathcal{M}_{P}} \sum_{p: a^{l} \in p} \sum_{q^{\prime}} m s^{m^{\prime}} z_{p, q^{\prime}}^{m^{\prime}}+\sum_{m^{\prime} \in \mathcal{M}_{N}} \sum_{p: a^{l} \in p} m s^{m^{\prime}} y_{p}^{m^{\prime}}+\right. \\
m f^{m}+\sum_{\substack{m^{\prime} \in \mathcal{M}_{P} \\
m^{\prime} \neq m}} \sum_{p} \sum_{q^{\prime}: a^{l} \in r\left(p, q^{\prime}\right)} m f^{m^{\prime}} z_{p, q^{\prime}}^{m^{\prime}}+ \\
\left.\sum_{k} \sum_{p: a^{l} \in p}\left(m^{k}+\sum_{t=t\left(i^{l}\right)}^{t(l(p))} f m_{t-t(f(p))}^{k}\right) x_{p}^{k}+\sum_{p: a^{l} \in p} w_{p}\right] \\
m \in \mathcal{M}_{P} \text {, path } q,
\end{gathered}
$$

where

$$
\Phi_{q, l}^{m}=\phi_{a^{l}}^{m} \prod_{l^{\prime}=l+1}^{|q|}\left(1-\phi_{a^{l^{\prime}}}^{m}\right) .
$$




\section{G. The Complete Model}

Since the cost to route commodities is negligible, we include only the refueling cost and the cost associated with elements. The objective function reads

$$
\min \sum_{m \in \mathcal{M}_{p}} c^{m} \sum_{\substack{p \\ f(p)=s}} \sum_{q} z_{p, g}^{m}+\sum_{m \in \mathcal{M}_{n}} c^{m} \sum_{p} y_{p}^{m}+f \sum_{p} w_{p}+f \sum_{m \in \mathcal{M}_{p}} m f^{m} \sum_{\substack{p \\ f(p)=s}} \sum_{q \neq \emptyset} z_{p, q}^{m},
$$

where $c^{m}$ is the cost of using element $m$ and $f$ is the per unit fuel cost. Note that $c^{m}$ should not include the fuel cost. The last term captures the fuel cost of the propulsive elements on their very first path originating at $s$ and assuming they burn (i.e. $q \neq \emptyset$ ). This is the fuel that is preloaded on the Earth into selected propulsive elements.

The model includes constraints (1) through (13). In addition, all $x$ and $w$ variables are nonnegative and all $z$ and $y$ variables are binary.

\section{Solution Methodology}

The model presented in the previous sections is complex and requires a sophisticated algorithm to be implemented in order to obtain good solutions. Due to the number of variables and constraint, in order to obtain good solutions quickly, heuristic optimization methods are employed to find good solutions.

The optimization of the interplanetary supply chain logistics problem has three components: commodity routing, commodity assignment to elements, and propulsive element to burn arc assignment. The commodity routing is performed first, since the entire architecture is driven by the commodity demand. Next, given the commodity paths through the network, the commodities are assigned to elements. Finally, since the mass of the elements and commodities are known for each arc in the network, the propulsive element assignment can be performed. The following section provides a detailed explanation of the algorithms employed.

\section{A. Heuristic Optimization}

As stated above, the commodity routing is performed first. The algorithm proceeds as follows. A commodity is selected and an auxiliary network is constructed such that all origin nodes for which the commodity is available are connected to a source node and all destination nodes for which the commodity can be delivered to are connected to a sink node. For every arc in the auxiliary network, a cost is assigned that is equivalent to the $\Delta V$ of the arc. The $\Delta V$ of an arc is chosen as the metric for cost in the auxiliary network since the amount of $\Delta V$ required drives the mass of the fuel and therefore the mass of the system. A shortest path with respect to $\Delta V$ from the source node to the sink node is found and when these fictitious nodes are removed, the path of the commodity is obtained. This procedure is repeated until all of the commodities have been routed on paths.

In reality, multiple commodities often travel on the same flight, and hence on the same path. To encourage multiple commodity assignments on the same paths, a fraction of the $\Delta V$ for an arc is subtracted for each arc already selected for a commodity path. Thus, after the first commodity is routed, each subsequent commodity routing receives a benefit for selecting previously chosen arcs for its path. Thus, although commodities are not required to share paths, they are encouraged to do so.

After the commodity paths are determined, the element to commodity assignment is performed. However, in order to perform this assignment, some preliminary manipulations are necessary. Since the network has arcs that only proceed forward in time, the nodes, and therefore arcs, can be arranged based on this order. This ordering is known as the topological order, and the details can be found in many network modeling books, such as Reference 4. A topological order of the nodes and arcs is necessary to ensure that all assignments on downstream connected arcs are determined prior to the current arc assignment.

Given the topological order, the arcs are selected and the total mass and volume of all commodities on the arc is determined. The element selection process proceeds as follows. The elements are ranked in order of preference, where the general preference is to have a low cost, high capacity element; however the exact weighting of cost and capacity are unknown. Thus, six different score functions have been created to rank the elements, and are provided in Equation 14. One of these six score functions is then selected uniformly at random and the elements are then ranked according to their score. The probability of an element being selected is determined by this score. Specifically, the probability of selection is determined by the ratio of 
the score of an element to the total score of the sum of all elements. Given this distribution, a random number is generated, and an element is selected if the random number falls into the interval corresponding to the element's probability of selection. The ranking process is repeated until all commodities are contained within elements. As the remaining arcs are selected, already selected elements are examined to determine if they can be used before selecting a new element using the above described ranking. At the end of this process all elements used to house commodities have been determined, as well as their paths through the network.

$$
\begin{aligned}
& S_{1}=\frac{\text { Cost }}{\text { ComMass }} \quad S_{2}=\frac{\text { Cost }^{2}}{\text { ComMass }} \\
& S_{3}=\text { Cost } \quad S_{4}=\frac{\sqrt{\text { Cost }}}{\text { ComMass }} \\
& S_{5}=\frac{\text { Cost }}{\text { ComMass }^{2}} \quad S_{6}=\frac{\text { Cost }}{\sqrt{\text { ComMass }}}
\end{aligned}
$$

Finally, the element to burn arc assignment is conducted. Again, a topological order of the arcs is required, but since only burn arcs are necessary all waiting arcs are ignored in the topological order. Given an arc, an element to burn arc is assigned, based on the rocket equation, ${ }^{6}$ and the assignment proceeds as follows. If a propulsive element is already on the arc, a check is performed to determine if the fuel available in the element is enough to perform the burn, given the total amount of mass on the arc. An element is already on the arc if it is holding commodity mass or if it was used on a consecutive burn arc and there is remaining fuel. If the element satisfies the rocket equation, then it is allocated to perform the burn and the amount of fuel required to do so is subtracted from the available fuel in the element. If not, then a new propulsive element is selected as follows. The elements are ranked using one of the six score function listed in Equation 15. The six score functions represent different weightings of low cost and high fuel mass. By selecting one of these score functions uniformly at random, the elements are evaluated and ranked, and an element is selected with a probability equivalent to the value of its rank in a similar manner as described above. This process is repeated until every burn arc has a propulsive element assigned to it.

$$
\begin{aligned}
S_{1} & =\frac{\text { Cost }}{\text { FuelMass }} & S_{2} & =\frac{\text { Cost }^{2}}{\text { FuelMass }} \\
S_{3} & =\text { Cost } & S_{4} & =\frac{\sqrt{\text { Cost }}}{\text { FuelMass }} \\
S_{5} & =\frac{\text { Cost }}{\text { FuelMass }^{2}} \quad S_{6} & = & \frac{\text { Cost }}{\sqrt{\text { FuelMass }}}
\end{aligned}
$$

Since, this is a heuristic randomized algorithm these steps are repeated many times. At the end of each iteration, the current solution is checked to determine if it is the best solution obtained thus far. If so, the solution is saved. After the maximum number of iterations is reached, the best solution is returned. The overall algorithm is shown in Figure 5 .

\section{Lunar Outpost Scenario}

This model and solution methodology was applied to the mission design problem for developing a lunar outpost. The lunar outpost scenario represents a complex set of multiple space flights over the period of 3 years. The need to deliver commodities to develop the lunar outpost as well as resupply the base with crew provisions provides an example that shows the benefit of considering interplanetary logistics for mission planning.

A simplified version of the model is implemented to solve the lunar outpost example. Specifically, loss and gain factors are not considered for commodity paths. Additionally, the path lengths are not constrained by the maximum time that commodities can travel. To avoid extended travel times for crews, strict intervals are imposed on both the availability and delivery windows. Table 1 provides commodity information for the lunar outpost example. 


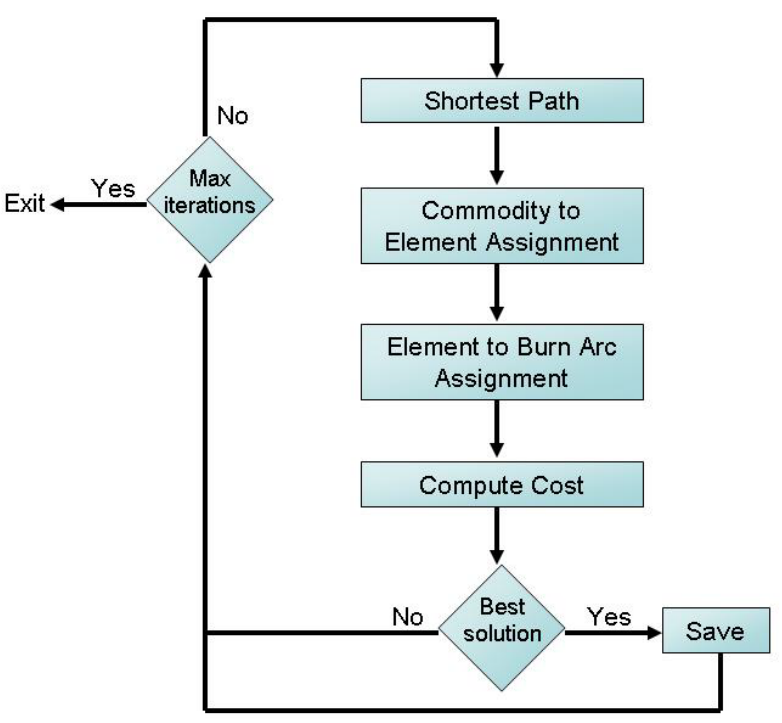

Figure 5. Flow Diagram of Heuristic Optimization

Table 1. List of Commodities and Properties for Lunar Outpost

\begin{tabular}{|c|c|c|c|c|c|c|c|}
\hline $\begin{array}{c}\text { Class of } \\
\text { Supply }\end{array}$ & Demand & $\begin{array}{c}\text { Starting } \\
\text { Node }\end{array}$ & $\begin{array}{c}\text { Time } \\
\text { Interval }\end{array}$ & $\begin{array}{c}\text { Ending } \\
\text { Node }\end{array}$ & $\begin{array}{c}\text { Time } \\
\text { Interval }\end{array}$ & $\begin{array}{c}\text { Mass } \\
(\mathrm{kg})\end{array}$ & $\begin{array}{c}\text { Volume } \\
\left(\mathrm{m}^{3}\right)\end{array}$ \\
\hline Operations & 100 & LEO & 1,1096 & LPS & 11,11 & 10 & 0.05 \\
\hline Provisions & 792 & LEO & 400,1096 & LPS & 466,466 & 100 & .07 \\
\hline Operations & 213 & LEO & 400,1096 & LPS & 466,466 & 10 & 0.05 \\
\hline Stowage & 56 & LEO & 400,1096 & LPS & 466,466 & 10 & 0.07 \\
\hline Exploration & 217 & LEO & 400,1096 & LPS & 466,466 & 10 & 0.05 \\
\hline Waste & 26 & LEO & 400,1096 & LPS & 466,466 & 10 & 0.05 \\
\hline Provisions & 67 & LEO & 400,1096 & LPS & 649,649 & 10 & 0.07 \\
\hline Provisions & 25 & LEO & 600,1096 & LPS & 748,748 & 10 & 0.07 \\
\hline Exploration & 25 & LEO & 600,1096 & LPS & 748,748 & 10 & 0.05 \\
\hline Crew & 4 & LEO & 740,1096 & LPS & 748,748 & 100 & 2 \\
\hline Provisions & 500 & LEO & 600,1096 & LPS & 831,831 & 10 & 0.07 \\
\hline Provisions & 185 & LEO & 600,1096 & LPS & 929,929 & 10 & 0.07 \\
\hline Crew & 4 & LEO & 900,1096 & LPS & 929,929 & 100 & 2 \\
\hline Provisions & 500 & LEO & 600,1096 & LPS & 1014,1014 & 10 & 0.07 \\
\hline
\end{tabular}


In order to transport the commodities from Low Earth Orbit (LEO) to the lunar polar surface (LPS) the properties of the available elements must be provided. Table 2 provides the element properties for the lunar outpost example.

Table 2. List of Elements and Properties for Lunar Outpost

\begin{tabular}{|c|c|c|c|c|c|c|c|}
\hline $\begin{array}{l}\text { Element } \\
\text { Type }\end{array}$ & $\begin{array}{c}\text { Fuel } \\
\text { Mass (kg) }\end{array}$ & $\begin{array}{l}\text { Isp } \\
(\mathrm{sec})\end{array}$ & $\begin{array}{l}\text { Structural } \\
\text { Mass (kg) }\end{array}$ & $\begin{array}{c}\text { Mass } \\
\text { Capacity (kg) }\end{array}$ & $\begin{array}{c}\text { Volume } \\
\text { Capacity }\left(\mathrm{m}^{3}\right)\end{array}$ & $\begin{array}{l}\text { Number } \\
\text { Available }\end{array}$ & Cost \\
\hline CaLV 1st Stage & 1785198 & 296 & 200704 & 0 & 0 & 10 & 1 \\
\hline CaLV 2nd Stage & 819792 & 452 & 97641 & 0 & 999 & 10 & 1 \\
\hline LSAM Descent Stage & 28918 & 440 & 6137 & 350 & 5 & 10 & 1 \\
\hline EDS & 240000 & 452 & 20011 & 0 & 0 & 10 & 1 \\
\hline LSAM Cargo & 0 & 0 & 1000 & 15000 & 50 & 10 & 1 \\
\hline LSAM Ascent Stage & 5863 & 362 & 5128 & 1850 & 1 & 10 & 1 \\
\hline CLV Boost Stage & 504511 & 269 & 81828 & 0 & 0 & 10 & 1 \\
\hline CLV Upper Stage & 163529 & 452 & 17507 & 0 & 0 & 10 & 1 \\
\hline Lunar CEV CM & 0 & 0 & 8034 & 500 & 1 & 10 & 1 \\
\hline Lunar CEV SM & 11657 & 362 & 3997 & 0 & 0 & 10 & 1 \\
\hline
\end{tabular}

The time expanded network consists of three static nodes: Low Earth Orbit (LEO), Lunar Polar Low Orbit (LPLO), and Lunar Polar Surface (LPS). The time horizon is 3 years long and is discretized by the day. Using the commodities provided in Table 1 and the elements given in Table 2, the optimization methodology described above was employed to determine the solution depicted in Figures 6, 7, and 8.

Figures 6, 17, and 8] show the evolution in time of the transportation of commodities and elements through the time expanded network. If we examine these figures, we see that the first flight delivers only the first Crew Operations commodity, since the delivery time is much earlier than all of the other commodities. The next six commodities leave LEO on one flight; however the sixth commodity (Crew Provisions) must be delivered at a later time and therefore waits in LPLO as the other commodities are delivered to the lunar surface. This waiting requires that an additional propulsive element be delivered to LPLO to transport the remaining commodity to the surface. The next flight consists of five different commodities with three different delivery dates. Again, additional propulsive elements must be sent to ensure that the commodities waiting in LPLO have the propulsive capability to reach the lunar surface on the expected delivery date. A final flight from LEO occurs during this interval that directly delivers crew to the surface.

This example demonstrates a few interesting decisions made by the optimizer. First, since all elements have a cost of one, the objective is simply to minimize the number of elements required. Notice that the LSAM Descent Stage (LSAM DS) and the LSAM Ascent Stage (LSAM AS) are both selected as the propulsive elements for lunar orbit injection and descent burns. As stated before, no restriction on the use of elements on a given arc is defined and therefore either element can be used for descent. On the third flight leaving LEO, at time 741 (Figure 7), a single Lunar CEV Command Module (CEV CM) is selected. Although this implies that this selection occurs because of the shipment of crew, this selection is arbitrary since crew are treated as any other commodity, as can be demonstrated by examining the final flight where the crew are transported to the lunar surface in the LSAM Cargo Element. In reality, crewed flights require special elements; however, implementing these requirements is beyond the scope of this model and must be handled by a post-optimality decision analysis.

\section{Conclusion}

In order for space exploration to be sustainable, interplanetary logistics must be considered during mission planning. Research conducted in the terrestrial logistics and operations research communities provides a wealth of modeling tools and solution approaches that can be extended to enable interplanetary logistics decisions. This paper explores the requirements necessary to define the interplanetary logistics problem and extends a modeling tool traditionally utilized in terrestrial logistics to incorporate the astrodynamic relationships of space travel. Using the time expanded network as a decision framework, a complex math- 


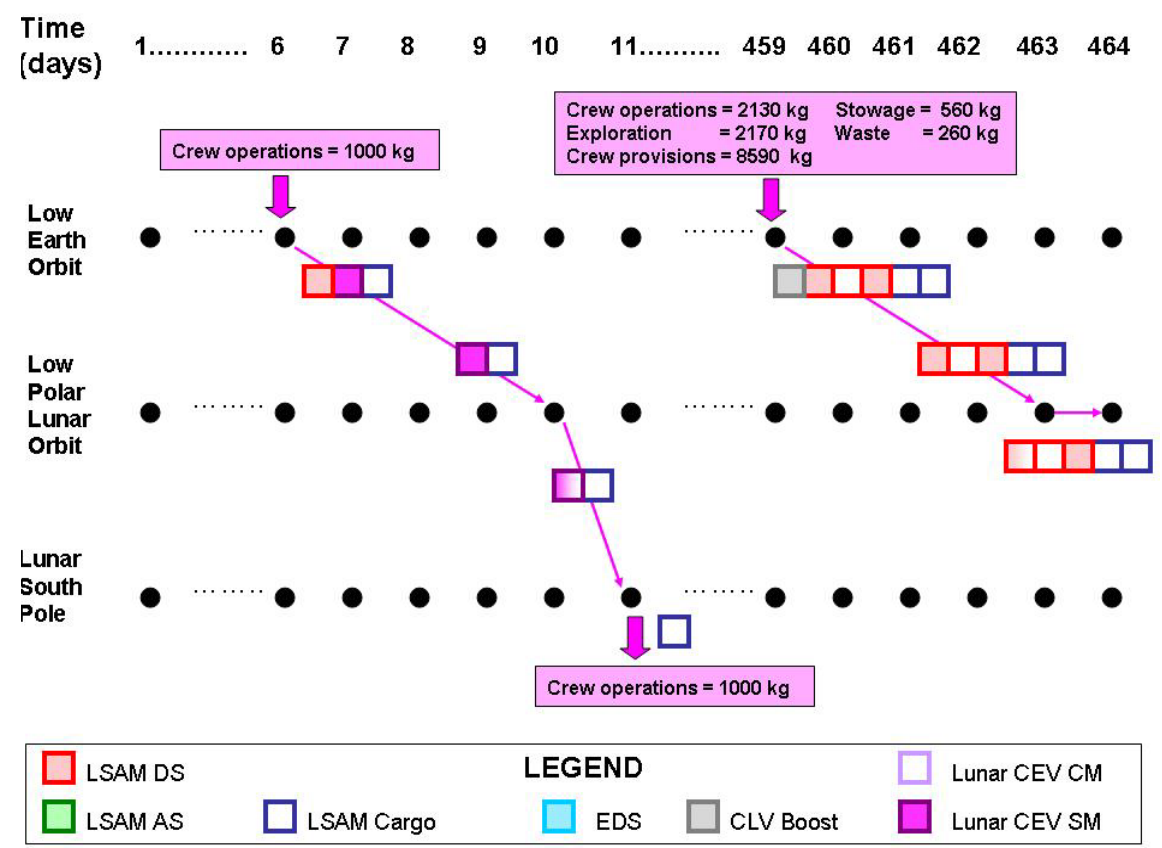

Figure 6. Lunar Outpost Example

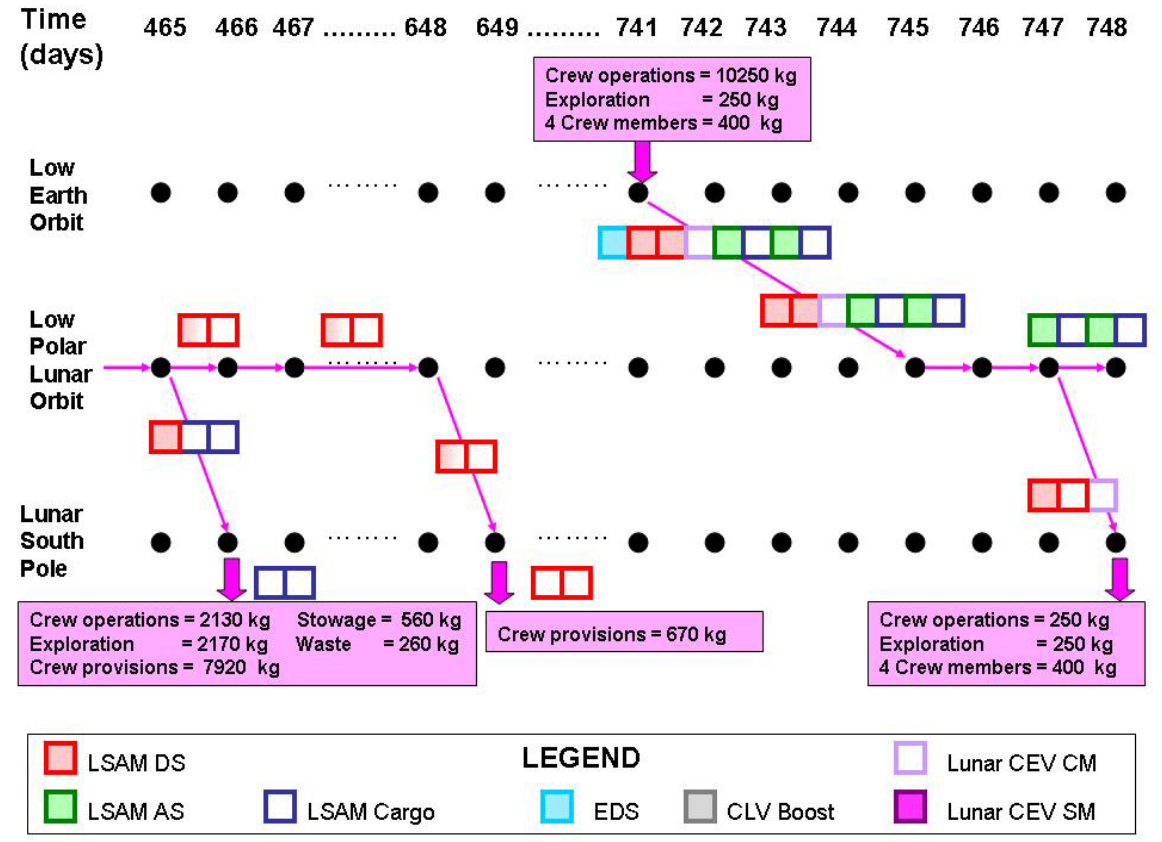

Figure 7. Lunar Outpost Example Continued 


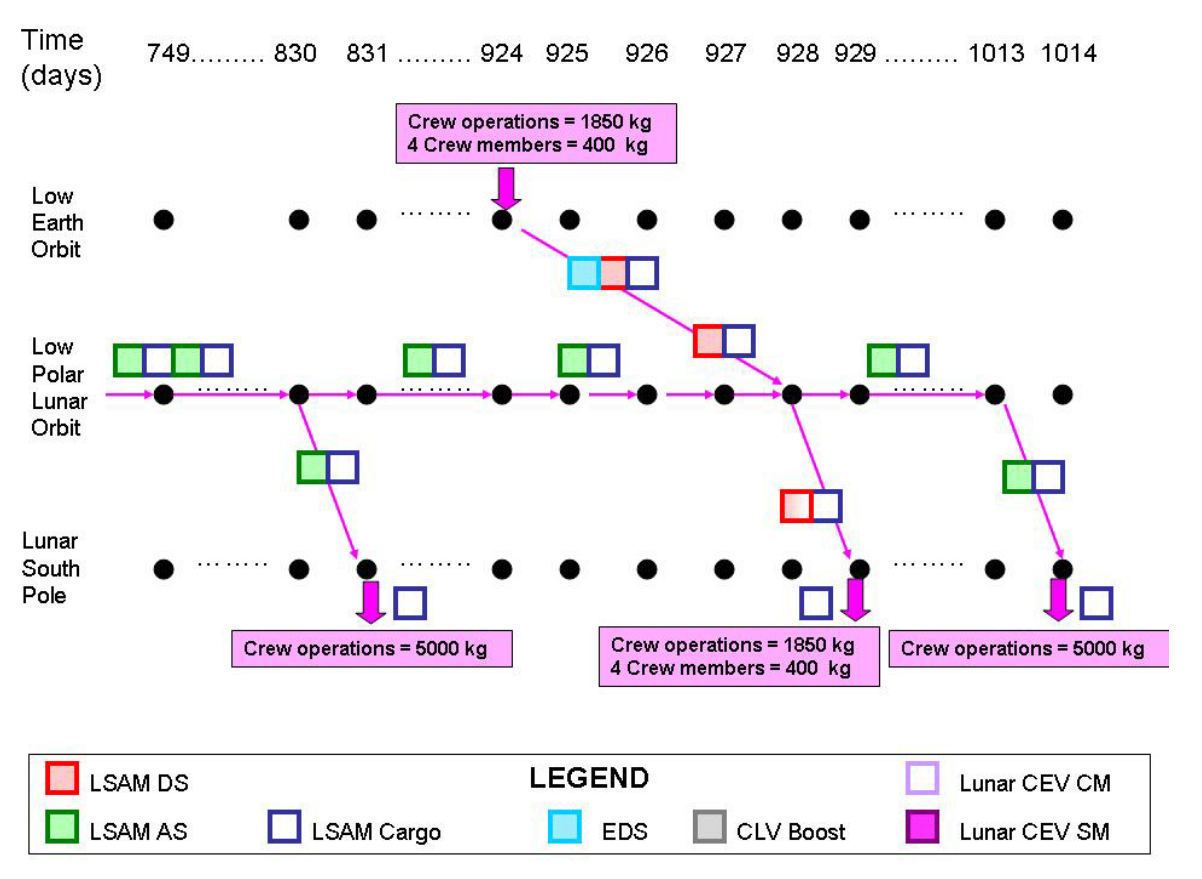

Figure 8. Lunar Outpost Example Concluded

ematical model was developed to incorporate the fundamental constraints of in-space transportation. Due to modeling complexities and problem size, a heuristic optimization algorithm was developed to explore the design space and find good solutions to the complex problem. This methodology was demonstrated for the example of a lunar outpost scenario, where the benefits of considering the interaction of multiple missions was seen in the reduction of the number of elements required to transport all of the commodities to the lunar surface, as compared to only direct flights to the surface.

Although the model is comprehensive, the solution approach can be improved to produce better solutions. Specifically, mission returns have yet to be implemented in this framework. In addition, some of the commodity parameters such as the loss/gain factor have yet to be utilized. Finally, the design space can be enlarged by incorporating low thrust propulsive elements; however the coupling between the trajectories and the element properties needs to be disentangled.

\section{References}

\footnotetext{
${ }^{1}$ Bush, P. G. W., "A Renewed Spirit of Discovery: A President's Vision for U.S. Space Exploration," Speech given on January 14, 2004.

${ }^{2}$ David Simchi-Levi, Julien Bramel, X. C., The logic of logistics: theory, algorithms, and applications for logistics and supply chain management, Springer, 2005.

${ }^{3}$ Yang, L. and Kornfeld, R., "Examiniation of the Hub-and-Spoke Network: A Case Example Using Overnight Package Delivery," 41st Aerospace Sciences Meeting and Exhibit, AIAA, 2003.

${ }^{4}$ Ravindra Ahuja, Thomas Magnanti, J. O., Network Optimization, Prentice Hall, 1993.

${ }^{5}$ Chan, L., M. A. S.-L. D., "Uncapacitated Production/Distribution Planning Problems with Piece-wise Linear Concave Costs," .

${ }^{6}$ Battin, R. H., An Introduction to the Mathematics and Methods of Astrodynamics, Revised Edition, AIAA Education Series, 1999
} 